Research Paper

\title{
Feasibility and Efficacy of Simultaneous Integrated Boost Intensity-modulated Radiation Therapy based on MRI-CT fusion in Patients with Brain Metastases of Non-small Cell Lung Cancer
}

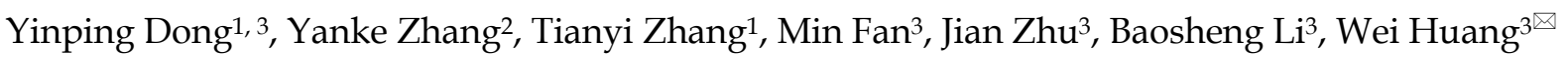 \\ 1. School of Medicine and Life Sciences, University of Jinan-Shandong Academy of Medical Sciences, Jinan, China; \\ 2. Department of Radiation Oncology, the General Hospital of Yan-zhou Mining Group, Jining, China; \\ 3. Department of Radiation Oncology, Shandong Cancer Hospital affiliated to Shandong University, Shandong Academy of Medical Sciences, Jinan, China \\ $\triangle$ Corresponding author: Wei Huang, Fax: (+86)531-67626162; Tel: (+86)531-67626162; Address: 440 Jiyan Road, Jinan 250117, Shandong Province, China; \\ E-mail: alvinbird@163.com \\ (c) Ivyspring International Publisher. This is an open access article distributed under the terms of the Creative Commons Attribution (CC BY-NC) license \\ (https://creativecommons.org/licenses/by-nc/4.0/). See http://ivyspring.com/terms for full terms and conditions.
}

Received: 2018.04.08; Accepted: 2018.09.09; Published: 2018.10.31

\begin{abstract}
Purpose: To assess the feasibility and therapeutic effects of simultaneous integrated boost intensity-modulated radiation therapy (SIB-IMRT) based on the fusion imaging of magnetic resonance imaging (MRI) and computed tomography $(C T)$ as a dose-intensive technique in patients with brain metastases (BM) of non-small cell lung cancer (NSCLC).

Methods and materials: Forty-six NSCLC patients with 1 to 7 brain metastases were enrolled in this retrospective study between November, 2011 and February, 2017. Thirty-one patients $(67.4 \%)$ had 1-3 metastases (oligometastases), otherwise, more than 3 metastases were seen in only 15 patients (32.6\%). GTV (Gross tumor volume) contouring was based on the fusion imaging of MRI-CT, WBRT was prescribed in $37.5 \mathrm{~Gy} / 15$ fractions with a simultaneous boost in the metastatic lesions of $52.5 \mathrm{~Gy} / 15$ fractions.

Results: The median overall survival (OS) and intracranial progression free survival (PFS) for all the patients were 20.0 months and 11.0 months, respectively. The 6-month and 1 -year OS were $87.0 \%$ and $69.6 \%$ respectively, while the 6 -month and 1 -year PFS were $78.3 \%$ and $43.5 \%$ respectively. Until the end of the follow-up, 16 patients (34.8\%) were alive. No evidence of intracranial progress or recurrence was found in 6 patients (13.0\%) during the follow-up.

Conclusion: SIB-IMRT with the dose/fractionation based on the fusion imaging of MRI-CT is feasible and safe. It is beneficial to the NSCLC patients with BM and can reduce the overall costs of treatment.
\end{abstract}

Key words: non-small cell lung cancer (NSCLC), simultaneous integrated boost (SIB), intensity-modulated radiation therapy (IMRT), brain metastases (BM), magnetic resonance imaging (MRI)

\section{Introduction}

Lung cancer is currently the leading cause of cancer-related deaths worldwide [1] with non-small cell lung cancer (NSCLC) accounting for approximately $85 \%$ of all lung cancer cases [2]. Brain metastasis (BM) is a common complication in NSCLC patients. Not only deterioration of primary lesions and metastases, but brain metastases are the main cause of morbidity and mortality in NSCLC patients. Evidence comes that BM occurred in up to more than $10 \%$ patients with NSCLC at the time of diagnosis [3, 4], while $30 \%-40 \%$ of NSCLC patients develop brain metastasis at some point during the course of the 
disease [5].

The whole brain radiotherapy (WBRT) is used as a significant palliative modality of treatment in patients of multiple-lesion BM because most chemotherapeutic regimens are not optional due to the block of the blood-brain barrier. Concerning dose-fraction mode, 30 Gy in 10 fractions or 37.5 Gy in 15 fractions is recommended as standard treatment; meanwhile, 20 Gy in 5 fractions is an alternative for poor performers. By using WBRT, 4-6 months prolonged overall survival (OS) has obtained [6, 7]. However, patients simply received WBRT are prone to have intracerebral recurrence. Randomized studies approve that the use of WBRT combined with operation or additional stereotactic radiosurgery (SRS) or 3-dimentional conformal radiotherapy (3DCRT) for those with single or regional multiple lesions ( $<3$ metastatic lesions) of $\mathrm{BM}$ are more beneficial in the local control and median OS of nervous system than the use of only one method [8-10].

There is less chance for them to undergo palliative resection, and therefore, the successive SRS followed by WBRT is universally accepted for its promising therapeutic effect. Nevertheless, these two different radiation modes need their own treatment plans respectively, and there are more difficulties on plans connection, dose-fraction calculation and time intervals [11, 12]. Furthermore, inadequate redistribution and reoxygenation of SRS in biological effect, as well as the increase of total treatment period can impair local control in some degree, especially for certain subtype tumors in rapid proliferation [13]. The technique of simultaneous integrated boost intensity-modulated radiotherapy (SIB-IMRT) has well developed for years, which allows delivering different dosage simultaneously at a single fraction to different target volumes [14]. SIB-IMRT makes it possible to enhance the radiation intensity of corresponding volumes meanwhile not to extend the overall therapeutic period $[15,16]$.

We carried out a retrospective study, aiming to assess the feasibility and safety of SIB-IMRT as a dose-intensive technique for BM patients of lung cancer. Treatment response and survival of SIB-IMRT were also investigated in the paper.

\section{Patients and Methods}

\section{Patients' selection}

All patients were diagnosed with NSCLC by pathology or cytology. Patients had to be present with pathologically confirmed NSCLC and had clinical and radiological evidence of 1 to $7 \mathrm{BMs}$, which was clarified by enhanced magnetic resonance imaging
(MRI) scans, and inaccessible for surgery or chemotherapy; Karnofsky performance score (KPS) $\geq$ 60 ; life expectancy $\geq 1$ months; age $>18$ years; at least one lesion that can be measured before radiotherapy; adequate hematological function (neutrophil count $\geq$ $1.5 \times 10^{9} / \mathrm{L}$; hemoglobin $\geq 90 \mathrm{~g} / \mathrm{L}$; thrombocyte count $\geq 100 \times 10^{9} / \mathrm{L}$ ). Exclusion criteria were as follows: intracranial surgery history; complete loss of either locomotive or sensory functions; severe disturbance of consciousness (stupor or coma); psychologically diagnosed disorders or other severe chronic diseases, such as uncontrollable diabetes, significant cardiac disease and hypertension; pregnant and lactating female patients. All eligible patients delivered literal (written informed) consent, and the study was approved by the institutional review boards of the Shandong Cancer Prevention and Treatment Research Ethics Committee.

\section{Treatment details}

All the patients were immobilized by thermoplastic noninvasive mask and then underwent the helical CT (Philips Brilliance Big Bores CT) scanning in supine position, $3 \mathrm{~mm}$ a layer, and all the images were uploaded to treatment planning system (TPS, Eclipse 8.6, Varian Medical System, Palo Alto, CA). As a pre-radiotherapy, all patients underwent a contrast MRI scan, and the images of these MRI and the treatment planning CT scan were fused to help with optimum delineation.

Guided by the fusion images, we then delineated the gross tumor volume (GTV) based on the MRI scan. GTV was defined as the scope of visible intracranial lesions. And $\mathrm{PTV}_{1}$ (planning target volume) was generated by adding a $5 \mathrm{~mm}$ margin to the GTV. As well, the target volume of the whole brain was defined as $\mathrm{PTV}_{2}$. Prescription dose to $\mathrm{PTV}_{1}$ was 3.5 Gy per fraction to a total dose of 52.5Gy in 15 fractions. The $\mathrm{PTV}_{2}$ was delivered in daily 2.5 Gy fractions to a total of 37.5Gy in same fractions. All fractions were delivered five times a week. The treatment plans were designed to cover at least $95 \%$ of $\mathrm{PTV}_{1}$ with $95 \%$ isodose, within the dose ranging from $90 \%$ to $105 \%$. The dose constraints of organs at risk (OARs), namely, included the maximal doses (Dmax) of brain stem $\leq 54$ Gy, spinal cord $\leq 45 \mathrm{~Gy}$, optic nerve $\leq 54 \mathrm{~Gy}$, lens $\leq 9$ Gy and the mean dose of eye (Dmean) $\leq 35 \mathrm{~Gy}$, parotid $\leq 26$ Gy [17].

According to the linear quadratic formula, the biological equivalent dose (BED) could be estimated via $-B E D=n d \times[1+d /(a / \beta)]$, in which $n$ stands for the total number of prescribed dose; $d$ for the dose of each fraction; $(a / \beta)=10$ for acute effect [18]. 


\section{Adverse effect assessment and statistical analysis}

The final adverse effects were defined as the most severe side reaction from the first day of the treatment and 4 weeks post-radiotherapy, being scored via National Cancer Institute Common Toxicity Criteria for Adverse Events (NCI CTC-AE) version 3.0. Late toxicities were estimated through Radiation Therapy Oncology Group (RTOG) and European Organization for Research and Treatment of Cancer (EORTC) late radiation morbidity scoring schema after 3 months treatment. During the follow-up, all the patients should receive contrastenhanced MRI or CT for head to evaluate the therapeutic effect. The treatment effects were referred to Response Evaluation Criteria in Solid Tumors (RECIST 1.1). The clinical evaluations and the follow-up were at 3-month intervals up to the first 2 years. And thereafter, it was 6 months.

The main endpoint of the research was grade 3 or more severe neural toxicities. The other endpoints were overall survival, intracranial progress-free survival (PFS). OS was recorded from the initiation of the treatment to the death of subjects or the latest follow-up. PFS was also recorded from treatment start, and ended in cranial progression, patients' death or last follow-up. The response based on the MRI or CT about the brain lesions were classified as follows: (1) Progressive disease (PD) denoting increase in the number or in contrast enhancement of the original lesions; (2) stable disease (SD) that signified no apparent change in either the number or enhancement characteristics; (3) complete response (CR) that reflected both, reduction in number and/or enhancement pattern.

Kaplan-Meier method was used to assess the OS and PFS, and SPSS software pack (version 17.0, SPSS Inc, Chicago, IL) was employed for statistics analysis.

\section{Results}

\section{Patient characteristics}

Of all 52 patients, 46 met the inclusion criteria and were involved from Shandong Cancer Hospital \& Institute between November, 2011 and February, 2017. Ineligible ones were excluded for severe systematic disease $(n=2)$, incomplete study data $(n=$ $1)$, and lose of follow-up ( $n=3)$. The vast majority (31, $67.4 \%$ ) of these patients have 1-3 metastases (oligometastases) with more than three metastases seen in only $32.6 \%$ (15) of the patients. Of these, there was 15 with a single (solitary) metastasis, meanwhile, 8 had $>4$ brain metastases. All the eligible 46 patients received cranial radiotherapy, in which 7 patients were squamous cell carcinoma, 37 were adenocarcin- oma, and 2 were mixed cell carcinoma. The median age was 60 years (range, 34 - 81 years). The detailed baseline data of the subjects were seen in Table 1.

Table 1. Patient characteristics

\begin{tabular}{lll}
\hline Characteristics & Number of cases & $\%$ \\
\hline $\begin{array}{l}\text { Median age (range) } \\
\text { Gender }\end{array}$ & $60(34-81)$ & \\
Male & 27 & $58.7 \%$ \\
Female & 19 & $41.3 \%$ \\
Kanofsky performance status & & \\
90 & 8 & $17.4 \%$ \\
80 & 25 & $54.3 \%$ \\
70 & 9 & $19.6 \%$ \\
60 & 4 & $8.7 \%$ \\
No. of brain metastasis & & \\
1 & 15 & $32.6 \%$ \\
$2-3$ & 16 & $34.8 \%$ \\
$\geq 4$ & 15 & $32.6 \%$ \\
Location of brain metastasis & & \\
Supratentorial & 33 & $71.7 \%$ \\
Infratentorial & 7 & $15.2 \%$ \\
Both supra and infratentorial & 6 & $13.1 \%$ \\
Histopathological subtype & & $15.2 \%$ \\
Squamous cell carcinoma & 7 & $80.4 \%$ \\
Adenocarcinoma & 37 & $4.4 \%$ \\
Mixed cell carcinoma & 2 & \\
\hline
\end{tabular}

\section{Treatment characteristics}

The radiotherapy plan was revised before being performed. And the dose of OARs was well restrained. No more than $5 \%$ target volume received $110 \%$ prescribed dose. The BED of PTV 1 in SIB-IMRT was 59.06Gy; for $\mathrm{PTV}_{2}$ the BED was 39.06Gy. The parameters of the OARs were assessed via DVH (Table 2). As the primary endpoint of this study was "neurotoxicity", by application of $(a / \beta)=30$, which reflect the late effect of irradiation, the BED of $\mathrm{PTV}_{1}$ in SIB-IMRT was 113.75Gy (52.5Gy/15 fractions); for $\mathrm{PTV}_{2}$ the BED was 68.75Gy (37.5Gy / 15 fractions).

\section{Toxicity}

Acute treatment-related toxicities were detailed in Table 3. The most common adverse reactions were headache $(\mathrm{n}=24,52.2 \%)$, nausea $(\mathrm{n}=18,39.1 \%)$, dizziness $(\mathrm{n}=19,41.3 \%)$ and fatigue $17(\mathrm{n}=17$, $37.0 \%$ ). Radiotherapy was well tolerated and no grade 4 events, brain hernia, or intratumoral hemorrhage occurred. 2 patients had grade III nausea, and 2 patients got grade III vomiting. 2 had grade III headache, and 1 had grade III fatigue. Patients with grade II or III reaction received necessary treatment such as administration of mannitol and corticosteroids to alleviate the symptoms induced by high intracranial pressure. During or after radiotherapy, all had alopecia in different degree. Neither discontinued treatment, nor death caused by treatment was observed in the patients. 
We evaluated the neurocognitive function for all patients by use of MMSE (Mini-Mental State Examination) scores at the diagnosis of the BMs, 3 months after the radiotherapy (WBRT-IMRT), then every three months until the end of the PFS and the follow-up. The data of the MMSE were as follows in Table 4. The neurotoxicity included headache, dizziness, and blurred vision, memory loss, hearing loss, inattention and emotional dysfunction, even neurologic sequelae such as dementia, abulia, stupor, and coma. For long-term adverse event, four patients complained about the mild memory loss at least 6 months after treatment. Two patients showed deterioration of hearing nearly 1 year after treatment. During the follow-up, there also were 2 with the symptoms of inattention and emotional dysfunction, and 3 with coma towards the end of the follow-up. Symptoms existing before treatment such as headache, dizziness, and blurred vision were acutely exacerbated after the radiotherapy, especially after 3 months of the radiotherapy, but obviously ameliorated as time passes. But the irreversible neurotoxic symptom, such as memory loss, hearing loss and so on, which were considered as the late adverse effects, may gradually emerged and aggravated towards the end of life.

\section{Response evaluation}

For each patient, iconographical examination was served as evaluating the therapeutic response 4 weeks after radiotherapy. According to RECIST criteria, of the 46 patients, 3 patients had complete response (CR), 31 ones got partial response (PR), and the rest showed stable disease (SD). The overall response $(\mathrm{CR}+\mathrm{PR})$ rate was $73.9 \%$, and the disease control $(\mathrm{CR}+\mathrm{PR}+\mathrm{SD})$ rate was $100 \%$. No one got progressive disease $(\mathrm{PD})$ or died before the treatment response evaluation (Table 5).

\section{Survival}

The median follow-up time of these 46 patients who completed the planned therapy was 16.5 months (range: 1.5 - 60 months). In the meantime, the median survival and intracranial PFS of all the patients were 20.0 months (95\% CI: 15.4 - 24.6 months) and 11.0 months (95\% CI: 9.6 - 12.4 months), respectively. The OS and PFS were shown in Figure 1 and Figure 2. The 6-month and 1-year OS rate were $87.0 \%$ and $69.6 \%$ respectively, while the 6-month and 1-year PFS rate were $78.3 \%$ and $43.5 \%$ respectively. Until the last follow-up, 16 patients (34.8\%) were alive. Deterioration of extracranial lesion or systemic status was the main cause of the death $(\mathrm{n}=13)$. Among all patients, 12 patients were with extracranial lesions at the diagnosis of BMs but not all died from this, which included 7 bone metastases, 3 adrenal gland metastases and 2 ampulla area occupied lesions were included. Cranial progression and central nervous system (CNS) complications were also the reasons for the death $(n=5)$. Still 3 patients died of pulmonary infectious complications. There are a portion of patients who have developed a progression but not died $(n=10)$, however, no evidence of intracranial progress or recurrence was also found in 6 patients $(13.0 \%)$ during the follow-up.

Table 2. The DVH-based parameters of the OARs in radiotherapy

\begin{tabular}{lll}
\hline Organs & & mean \pm SD (cGy) \\
\hline Brain stem & Dmax & $4281 \pm 699$ \\
Spinal cord & Dmax & $3257 \pm 752$ \\
L. optic nerve & Dmax & $3700 \pm 708$ \\
R. optic nerve & Dmax & $3770 \pm 688$ \\
L. lens & Dmax & $507 \pm 166$ \\
& Dmean & $455 \pm 167$ \\
R. lens & Dmax & $515 \pm 173$ \\
& Dmean & $460 \pm 171$ \\
L. eye & Dmean & $911 \pm 456$ \\
R. eye & Dmean & $916 \pm 426$ \\
L. parotid & Dmean & $800 \pm 479$ \\
R. parotid & Dmean & $724 \pm 378$ \\
\hline
\end{tabular}

Table 3. Toxicity and adverse reaction

\begin{tabular}{llllll}
\hline $\begin{array}{l}\text { Adverse event } \\
\text { (grade) }\end{array}$ & $\mathbf{0}$ & I & II & III & $\begin{array}{l}\text { Total } \\
\text { (I+II+III) }\end{array}$ \\
\hline Dizziness & 27 & 12 & 7 & 0 & $19(41.3 \%)$ \\
Headache & 22 & 13 & 9 & 2 & $24(52.2 \%)$ \\
Nausea & 28 & 9 & 7 & 2 & $18(39.1 \%)$ \\
Vomiting & 33 & 5 & 6 & 2 & $13(28.3 \%)$ \\
Fatigue & 29 & 10 & 6 & 1 & $17(37.0 \%)$ \\
Myelotoxicity & 38 & 6 & 2 & 0 & $8(17.4 \%)$ \\
\hline
\end{tabular}

Table 4. Changes in MMSE over time

\begin{tabular}{lllll}
\hline Scores & $\begin{array}{l}\text { At the } \\
\text { diagnosis } \\
\text { of the BMs } \\
(\mathbf{n}=\mathbf{4 6})\end{array}$ & $\begin{array}{l}\text { 3 months } \\
\text { after the } \\
\text { WBRT-IMRT } \\
(\mathbf{n}=\mathbf{4 6 )}\end{array}$ & $\begin{array}{l}\text { At the end } \\
\text { of the PFS } \\
(\mathbf{n}=\mathbf{4 6})\end{array}$ & $\begin{array}{l}\text { At the end } \\
\text { of the } \\
\text { follow-up } \\
(\mathbf{n}=\mathbf{4 6})\end{array}$ \\
\hline $15-24$ & $5(10.9 \%)$ & $11(23.9 \%)$ & $13(28.3 \%)$ & $12(26.1 \%)$ \\
$25-30$ & $41(89.1 \%)$ & $35(76.1 \%)$ & $32(69.5 \%)$ & $33(71.7 \%)$ \\
$\begin{array}{l}\text { Information } \\
\text { missing }\end{array}$ & 0 & 0 & $1(2.2 \%)$ & $1(2.2 \%)$
\end{tabular}

Abbreviations: MMSE=mini-mental state examination. Data are $\mathrm{n}(\%)$ unless otherwise stated.

Table 5. Therapeutic efficacy $(n=46)$

\begin{tabular}{lll}
\hline Response & Number of cases & $\%$ \\
\hline CR & 3 & $6.5 \%$ \\
PR & 31 & $67.4 \%$ \\
SD & 12 & $26.1 \%$ \\
PD & 0 & 0 \\
Overall response & 34 & $73.9 \%$ \\
Disease control & 46 & $100.0 \%$ \\
\hline
\end{tabular}

Abbreviations: $\mathrm{CR}$ = complete release; $\mathrm{PR}$ = partial response; $\mathrm{SD}$ = stable disease; $\mathrm{PD}=$ progressive disease . 


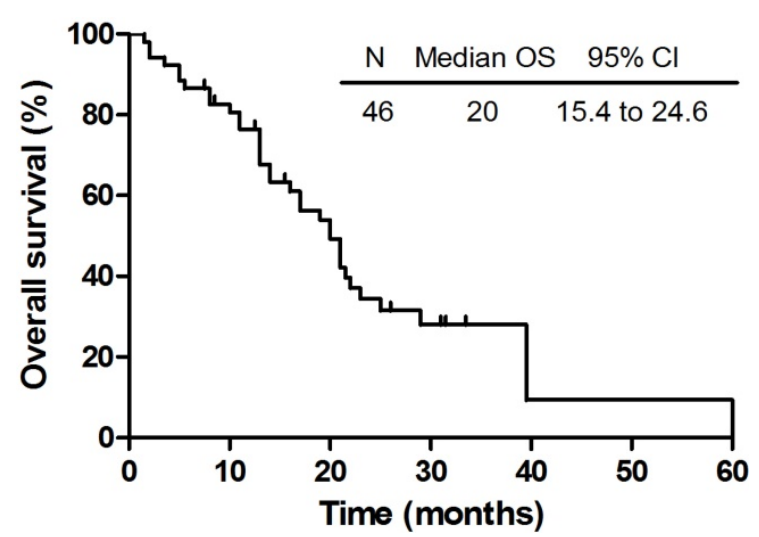

Figure 1. The OS of 46 patients who completed the planned therapy.

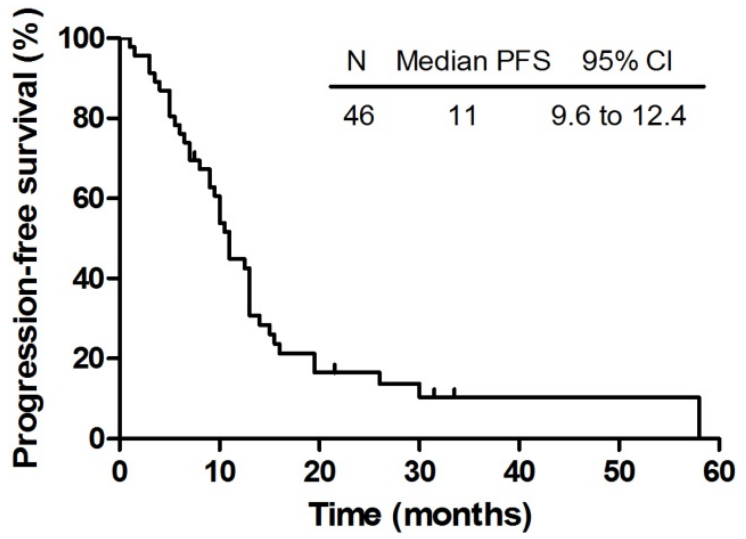

Figure 2. The PFS of 46 patients who completed the planned therapy.

\section{Discussion}

WBRT has played a crucial role as a main treatment in patients with intracranial metastases, and has been of great importance in prolonging progressfree survival (PFS) and improving QoL [19]. However, the total dose in 40 Gy is allowed in WBRT, otherwise, potential permanent neural injure will be caught in CNS. Bolgelt et al. found no difference of $20 \mathrm{~Gy} / 5$ fractions, $30 \mathrm{~Gy} / 10$ fractions, $30 \mathrm{~Gy} / 15$ fractions, 40 Gy/15 fractions and $40 \mathrm{~Gy} / 20$ fractions of intracranial radiation in median survival [20, 21]. National Comprehensive Cancer Network (NCCN) recommended the standard regimen of WBRT was $37.5 \mathrm{~Gy} / 15$ fractions or $30 \mathrm{~Gy} / 10$ fractions. Since the single use of operation does not have good disease control, especially for one BM; and local control failure in $35 \%-60 \%$ patients ensued $[9,22]$, therefore, resection or SRS plus WBRT were well discussed.

Whereas, it is controversial that the role of WBRT plays in NSCLC in the recently studies, which indicated that WBRT provided no clinically significant OS benefit, or needed for steroids over supportive care [23]. In the past decades, evidence comes that SRS alone is present with improved survival for patients with 1 to $3 \mathrm{BM}$ [24]. A recent multiinstitutional JLGK 0901 prospective study revealed the class- 2 evidence that SRS without WBRT is an effective treatment for patients up to 10 metastatic lesions. The data suggested that SRS can be used and WBRT can be withheld in selected patients with multiple lesions to avoid acute or chronic adverse effects, especially neurocognitive decline, without causing survival disadvantage [25]. However, patient selection is very critical for SRS treatment alone. Three phase 3 randomized trials (NCCTCG N0574, EORTC 22952-26001, and JROSG 99-1) have proved that compared to SRS, SRS combined with WBRT were more effective in local control and PFS for oligometastases (1-4 BM), the OS made no difference yet [10, 26, 27]. But, significantly better OS was observed in the diagnosis-specific Graded Prognostic Assessment (DS-GPA) 2.5-4.0 group in WBRT + SRS vs the SRS alone.

While in the aspect of planning, though RTOG 9508 confirmed that the delivery of SRS after WBRT did prolong the PFS [28], the overall treatment period was also extended. And evidence came that the treatment plan of simultaneous intensity boost in BM was more beneficial than that in the sequential radiotherapy. By using synchronously tomotherapy, Rodrigues et al. demonstrated $60 \mathrm{~Gy} / 10$ fractions intralesional boost with WBRT of $30 \mathrm{~Gy} / 10$ fractions was biologically equivalent with a stereotactic radiosurgery boost of $18 \mathrm{~Gy} / 1$ fraction with $30 \mathrm{~Gy} / 10$ fractions WBRT, and this modality generated no more treatment-relevant toxicities [29].

In our study, SIB-IMRT was utilized in the treatment of patients with BM. Not only oligometastases but also disseminated BMs which included gross and microscopic lesions could be controlled. Especially advocated in this study, harboring lesions of greater number (1-7) was included. WBRT was exerted in 37.5 Gy/15 fractions; meantime, a simultaneous boost in the metastatic lesions could reach 52.5 Gy in 15 fractions. This modality made dose of different PTVs computed in a single plan, and the mature technique of SIB-IMRT had its advantages in plan design and skill exertion, when comparing with other forms of synchronous boost radiation therapies; in the meantime, the dose in critical tissues and organs were still exactly confined within the limits. We finally obtained favorable outcomes, namely, $4.3 \%$ grade III nausea and vomiting respectively, overall response rate was $73.9 \%$, the 6-month and 1-year PFS rate were 78.3\% and $43.5 \%$. Notably, the sample size of the trial was small.

In the research of BM treatment, the balance of maximal therapeutic effects and minimal side effects 
was of paramount significance. WBRT may improve intracranial control by a control of microscopic disease and subclinical focus, but is associated with significant long-term detrimental effects on overall outcome, such as neurocognitive decline and delaying systemic therapy [30]. Weber et al. investigated that under the premise of no augmenting toxicity to CNS, higher dose radiation therapy in oligo-BM might favor the patients, especially when patients with relative good mood, such as younger age, higher performance score, fewer $\mathrm{BM}$ and good control of primary disease [31]. So, in this study, we chose 37.5 Gy / 15 fractions other than 30 Gy / 10 fractions as the standard therapeutic regimen in WBRT. The median OS and PFS were 20.0 and 11.0 months, respectively, and most acute treatment-relative toxicities had been well tolerated. No one stopped the therapy because of the toxicities. Grade III adverse reaction were observed in few patients and had been relieved by symptomatic treatment afterwards. As for long-term toxicities, 4 patients had slight memory loss and the other 2 suffered from hearing decline, which did not impact the QoL severely. Notably, the toxicities have no relationship with the number of BM lesions irradiated.

Another factor that made SIB-IMRT beneficial to our patients was its economic efficiency. Most recently, a clinical study by Tiwari et al. had also discussed the advantages of the technique [32], and they suggested the higher dose of boost in oligo BM during WBRT has more advantages than SRS in the aspect of simplifying procedure and reducing associated cost, which was more meaningful for developing countries.

In order to avoid or delay the need for WBRT or reduce the potential adverse effects with the use of WBRT, specialists try a lot of new methods to reach it. The potential of Hippocampal-avoidance WBRT has been proved in a phase 2 trial; nevertheless, a phase 3 trial should be carried out to verify its ability to preserve cognitive function. That the visible (or macroscopic) lesions can treated with SRS and microscopic lesions can be controlled by targeted therapy and immunotherapy as a standpoint springs up. When it comes to this, what is deserved to be mentioned is the study by Yi Long Wu et al., which pointed out that targeted therapy, Icotinib, was preferred with immproved intracranial progressionfree survival (iPFS), PFS and ORR over WBRT along with chemotherapy in advanced NSCLC with BM harboring epidermal growth factor receptor (EGFR) mutations[33].

To be concluded, the treatment of SIB-IMRT exerted in with 1 to $7 \mathrm{BMs}$ patients of NSCLC was well tolerated. The outcomes were promising and have proved that the SIB-IMRT is a feasible regimen. However, as a trial with small sample capacity, further randomized investigation and long-term observation of advanced neurological functions should be made to access the survival advantage of SIB-IMRT. Whether to use WBRT or not to control BM is still needed answering. How the RT should be given with targeted agents and/or immunotherapy which have both systemic and intracranial activity to the patients with drive gene is also needed further investigated.

\section{Abbreviations}

BM: Brain metastases; QoL: Quality of life; WBRT: Whole brain radiotherapy; OS: Overall survival; SRS: Stereotactic radiosurgery; 3DCRT: 3-dimentional conformal radiotherapy; SIB-IMRT: Simultaneous integrated boost intensity- modulated radiotherapy; CT: Computed tomography; MRI: Magnetic resonance imaging; KPS: Kanofsky performance score; TPS: Treatment planning system; GTV: Gross target volume; PTV: Planning target volume; OAR: Organ at risk; BED: Biological equivalent dose. CTC-AE: Common toxicity criteria for adverse events; RTOG: Radiation Therapy Oncology Group; EORTC: European Organization for Research and Treatment of Cancer; RECIST: Response evaluation criteria in solid tumors; PFS: Progress-free survival; CR: Complete release; PR: Partial release; SD: Stable disease; PD: Progressive disease; CNS: Central nervous system; iPFS: intracranial progression-free survival.

\section{Acknowledgements}

This work was supported by grant no.81402538, no.81530060, no.81671785 from the National Natural Science Foundation of China.

\section{Competing Interests}

The authors have declared that no competing interest exists.

\section{References}

1. Siegel RL, Miller KD, Jemal A. Cancer statistics, 2015. CA: a cancer journal for clinicians. 2015; 65: 5-29.

2. Park HS, Decker RH, Wilson LD, Yu JB. Prophylactic Cranial Irradiation for Patients With Locally Advanced Non-Small-Cell Lung Cancer at High Risk for Brain Metastases. Clinical lung cancer. 2015; 16: 292-7.

3. Eichler AF, Loeffler JS. Multidisciplinary management of brain metastases. The oncologist. 2007; 12: 884-98.

4. Langer CJ, Mehta MP. Current management of brain metastases, with a focus on systemic options. Journal of clinical oncology : official journal of the American Society of Clinical Oncology. 2005; 23: 6207-19.

5. Huang $Q$, Ouyang X. Predictive biochemical-markers for the development of brain metastases from lung cancer: clinical evidence and future directions. Cancer epidemiology. 2013; 37: 703-7.

6. Tsao MN, Lloyd NS, Wong RK, Rakovitch E, Chow E, Laperriere N, et al. Radiotherapeutic management of brain metastases: a systematic review and meta-analysis. Cancer treatment reviews. 2005; 31: 256-73.

7. Lemke DM. Epidemiology, diagnosis, and treatment of patients with metastatic cancer and high-grade gliomas of the central nervous system. 
Journal of infusion nursing : the official publication of the Infusion Nurses Society. 2004; 27: 263-9.

8. Patchell RA, Tibbs PA, Regine WF, Dempsey RJ, Mohiuddin M, Kryscio RJ, et al. Postoperative radiotherapy in the treatment of single metastases to the brain: a randomized trial. Jama. 1998; 280: 1485-9.

9. Li B, Yu J, Suntharalingam M, Kennedy AS, Amin PP, Chen Z, et al. Comparison of three treatment options for single brain metastasis from lung cancer. International journal of cancer. 2000; 90: 37-45.

10. Kocher M, Soffietti R, Abacioglu U, Villa S, Fauchon F, Baumert BG, et al. Adjuvant whole-brain radiotherapy versus observation after radiosurgery or surgical resection of one to three cerebral metastases: results of the EORTC 22952-26001 study. Journal of clinical oncology : official journal of the American Society of Clinical Oncology. 2011; 29: 134-41.

11. Hasegawa T, Kondziolka D, Flickinger JC, Germanwala A, Lunsford LD. Brain Metastases Treated with Radiosurgery Alone: An Alternative to Whole Brain Radiotherapy? Neurosurgery. 2003; 52: 1318-26.

12. Jyothirmayi R, Saran FH, Jalali R, Perks J, Warrington AP, Traish D, et al. Stereotactic Radiotherapy for Solitary Brain Metastases. Clinical Oncology. 2001; 13: 228-34

13. Bese NS, Hendry J, Jeremic B. Effects of prolongation of overall treatment time due to unplanned interruptions during radiotherapy of different tumor sites and practical methods for compensation. International journal of radiation oncology, biology, physics. 2007; 68: 654-61.

14. Orlandi E, Palazzi M, Pignoli E, Fallai C, Giostra A, Olmi P. Radiobiological basis and clinical results of the simultaneous integrated boost (SIB) in intensity modulated radiotherapy (IMRT) for head and neck cancer: A review. Critical reviews in oncology/hematology. 2010; 73: 111-25.

15. Franceschini D, Paiar F, Meattini I, Agresti B, Pasquetti EM, Greto D, et al. Simultaneous integrated boost-intensity-modulated radiotherapy in head and neck cancer. The Laryngoscope. 2013; 123: E97-103.

16. Wu $B$, McNutt $T$, Zahurak $M$, Simari $P$, Pang $D$, Taylor $R$, et al. Fully automated simultaneous integrated boosted-intensity modulated radiation therapy treatment planning is feasible for head-and-neck cancer: a prospective clinical study. International journal of radiation oncology, biology, physics. 2012; 84: e647-53.

17. Marks LB, Yorke ED, Jackson A, Ten Haken RK, Constine LS, Eisbruch A, et al. Use of normal tissue complication probability models in the clinic. International journal of radiation oncology, biology, physics. 2010; 76: S10-9.

18. Kocher M, Treuer H, Voges J, Hoevels M, Sturm V, Müller R-P. Computer simulation of cytotoxic and vascular effects of radiosurgery in solid and necrotic brain metastases. Radiotherapy and Oncology. 2000; 54: 149-56.

19. Sperduto PW, Berkey B, Gaspar LE, Mehta M, Curran W. A new prognostic index and comparison to three other indices for patients with brain metastases: an analysis of 1,960 patients in the RTOG database. International journal of radiation oncology, biology, physics. 2008; 70: 510-4

20. Borgelt B, Gelber R, Kramer S, Brady LW, Chang CH, Davis LW, et al. The palliation of brain metastases: Final results of the first two studies by the radiation therapy oncology group. International Journal of Radiation Oncology*Biology*Physics. 1980; 6: 1-9.

21. Borgelt B, Gelber R, Larson M, Hendrickson F, Griffin T, Roth R. Ultra-rapid high dose irradiation schedules for the palliation of brain metastases: Final results of the first two studies by the radiation therapy oncology group. International Journal of Radiation Oncology*Biology*Physics. 1981; 7: 1633-8.

22. Kaal ECA, Niël CGJH, Vecht CJ. Therapeutic management of brain metastasis. The Lancet Neurology. 2005; 4: 289-98.

23. Paula Mary Mulvenna MGN, Rachael Barton, Corinne Faivre-Finn, Paula Wilson, Barbara Moore, Elaine McColl, Iona Brisbane, David Ardron, Benjamin Sydes, Cheryl Pugh, Tanya Holt, Neil Bayman, Sally Morgan, Caroline Lee, Kathryn Waite, Richard Stephens, Mahesh M K Parmar, Ruth E Langley Whole brain radiotherapy for brain metastases from non-small lung cancer: Quality of life (QoL) and overall survival (OS) results from the UK Medical Research Council QUARTZ randomised clinical trial (ISRCTN 3826061). Journal of Clinical Oncology. 2015; 33: suppl.8005

24. Chang EL, Wefel JS, Hess KR, Allen PK, Lang FF, Kornguth DG, et al. Neurocognition in patients with brain metastases treated with radiosurgery or radiosurgery plus whole-brain irradiation: a randomised controlled trial. The Lancet Oncology. 2009; 10: 1037-44.

25. Yamamoto M, Serizawa T, Shuto T, Akabane A, Higuchi Y, Kawagishi J, et al. Stereotactic radiosurgery for patients with multiple brain metastases (JLGK0901): a multi-institutional prospective observational study. The Lancet Oncology. 2014; 15: 387-95.

26. Aoyama $\mathrm{H}$, Tago $\mathrm{M}$, Shirato $\mathrm{H}$, Japanese Radiation Oncology Study Group I. Stereotactic Radiosurgery With or Without Whole-Brain Radiotherapy for Brain Metastases: Secondary Analysis of the JROSG 99-1 Randomized Clinical Trial. JAMA oncology. 2015; 1: 457-64

27. Kim H, Rajagopalan MS, Beriwal S, Smith KJ. Cost-effectiveness Analysis of Stereotactic Radiosurgery Alone Versus Stereotactic Radiosurgery with Upfront Whole Brain Radiation Therapy for Brain Metastases. Clin Oncol (R Coll Radiol). 2017; 29: e157-e64.

28. Andrews DW, Scott CB, Sperduto PW, Flanders AE, Gaspar LE, Schell MC, et al. Whole brain radiation therapy with or without stereotactic radiosurgery boost for patients with one to three brain metastases: phase III results of the RTOG 9508 randomised trial. The Lancet. 2004; 363: 1665-72.
29. Rodrigues G, Yartsev S, Tay KY, Pond GR, Lagerwaard F, Bauman G. A phase II multi-institutional study assessing simultaneous in-field boost helical tomotherapy for 1-3 brain metastases. Radiation oncology. 2012; 7: 42.

30. Grossman SA, Ye X, Lesser G, Sloan A, Carraway H, Desideri S, et al Immunosuppression in patients with high-grade gliomas treated with radiation and temozolomide. Clinical cancer research : an official journal of the American Association for Cancer Research. 2011; 17: 5473-80.

31. Weber DC, Caparrotti F, Laouiti M, Malek K. Simultaneous in-field boost for patients with 1 to 4 brain metastasis/es treated with volumetric modulated arc therapy: a prospective study on quality-of-life. Radiation oncology. 2011; 6: 79.

32. Tiwari V, Pande SC, Verma K, Goel S. Simultaneous integrated boost with intensity modulated radiation therapy in brain oligometastases: A feasible technique for developing countries. South Asian journal of cancer. 2015; 4: $11-4$.

33. Wu YL, Yang JJ, Zhou C, Feng J, Lu S, Song Y, et al. PL03.05: BRAIN: A Phase III Trial Comparing WBI and Chemotherapy with Icotinib in NSCLC with Brain Metastases Harboring EGFR Mutations (CTONG 1201). Journal of Thoracic Oncology. 2017; 12: S6. 